\title{
Integración de Twitter y Facebook en la comunicación de la administración local: el Ayuntamiento de Elche como caso de estudio
}

Fernando Miguel Olabe | folabe@gmail.com

Universidad Miguel Hernández de Elche

José Antonio Márquez López| jose.marquez02@goumh.umh.es

Universidad Miguel Hernández de Elche

Palabras clave

"Ayuntamiento"; "Ciudadanía";

"Comunicación Local”; "Facebook”; "Gestión

comunicativa"; "Twitter".

Sumario

1. Introducción

2. Objetivos e hipótesis

3. Marco teórico

3.1 Las redes sociales en el ámbito de la

administración pública

4. Metodología

4.1 Variables analizadas en Twitter

4.2 Variables analizadas en Facebook

5. Resultados

5.1. Resultados del análisis en Twitter

5.2 Resultados del análisis en Facebook

6. Conclusiones

7. Bibliografía

\section{Resumen}

La incorporación de las redes sociales en las organizaciones públicas es un hecho. La administración pública ha comenzado a utilizar estas nuevas herramientas sociales del siglo XXI para establecer nuevas relaciones de confianza con los ciudadanos y proporcionar una información de valor y servicio para la audiencia. Sin embargo, la gestión de los medios sociales necesita de una atención continua a la actividad que se genera en los diferentes canales en red que existen en la actualidad. Las relaciones con los usuarios y la interacción con estos para conocer sus preocupaciones es una oportunidad para democratizar las instituciones a través del mundo digital. En este trabajo de investigación se ha realizado un análisis de contenido

sobre los perfiles en Twitter y Facebook del Ayuntamiento de Elche con el fin de conocer el tipo de gestión comunicativa realizada por la cuenta y valorar determinados aspectos como la presencia y relevancia de la cuenta, actividad de la misma, reacciones de los usuarios, además de la interacción y conversación entre emisores y receptores de la información.

La investigación muestra un comportamiento comunicativo unidireccional del Ayuntamiento de Elche en sus perfiles de Twitter y Facebook, que como consecuencia no generan interacción con los seguidores.

\section{Cómo citar este texto:}

Fernando Miguel Olabe, José Antonio Márquez López (2019): Integración de Twitter y Facebook en la comunicación de la administración local: el Ayuntamiento de Elche como caso de estudio, en Miguel Hernández Communication Journal, n¹0 (1), pp. 57 a 81. Universidad Miguel Hernández, UMH (Elche-Alicante). DOI: http://dx.doi.org/10.21134/mhcj.v10i0.275 


\section{Integration of Twitter and Facebook in the communication of the local administration: the City Council of Elche as a case study}

Fernando Miguel Olabe | folabe@gmail.com

Universidad Miguel Hernández de Elche

José Antonio Márquez López| jose.marquez02@goumh.umh.es Universidad Miguel Hernández de Elche

Keywords

"Citizenship"; "Communicative management";

"Facebook"; “Local Communication"; "Town hall”;

"Twitter".

Summary

1. Introduction

2. Aims and hypothesis

3. Theoretical frame

3.1 The social networks in the area of the public

administration

4. Methodology

4.1 Endpoints analyzed in Twitter

4.2 Endpoints analyzed in Facebook

5. Results

5.1. Results of the analysis in Twitter

5.2 Results of the analysis in Facebook

6. Conclusions

7. Bibliography

\begin{abstract}
The incorporation of social networks in the public organizations is a fact. Public administration has begun to use these new social tools of the 21 st century to establish new relationships of trust with citizens in order to provide valuable information and service to the audience. Nevertheless, social media management needs continuous attention about the activity of the different social channels that can be used nowadays. Relations and interaction with users to know their concerns is an opportunity to democratize institutions across the digital world.
\end{abstract}

In this research work will be studied the social media plan of Elche Town Hall in Facebook and Twitter in order to know what kind of management is done. This will allows the study of different endpoints such us the presence, relevance and activity of the account, users reactions and finally the interaction and conversations between issuer and receivers of the information.

The present investigation shows a communicative unidirectional behavior of the Town hall of Elche in his profiles of Twitter and Facebook, which as consequence do not generate interaction with the followers.

\section{How to cite this text:}

Fernando Miguel Olabe, José Antonio Márquez López (2019): Integration of Twitter and Facebook in the communication of the local administration: the City Council of Elche as a case study, in Miguel Hernández Communication Journal, nº10 (1), pp. 57 a 81. Universidad Miguel Hernández, UMH (Elche-Alicante). DOI: http://dx.doi.org/10.21134/mhcj.v10i0.275 


\section{Introducción}

La comunicación 2.0 ha supuesto una transformación en las relaciones entre las empresas e instituciones, tanto públicas como privadas, con sus respectivos públicos objetivos. Las redes sociales y las webs 2.0 son herramientas fundamentales en el nuevo paradigma de la comunicación, el cual requiere una mayor atención, capacidad de escucha y un seguimiento constante por parte de los emisores, con el fin de ofrecer al público un mejor servicio y generar una relación bidireccional que ayude a conseguir los objetivos de manera más rápida y eficaz (Herreros, 2008).

Las investigaciones previas que se han llevado a cabo en el ámbito de la comunicación en las administraciones públicas ponen el foco en el deber de las instituciones de trasladar a la población la información sobre la gestión que realizan. Esta función social sienta las bases de un modelo comunicativo que ayuda al ciudadano a resolver cualquier tipo de duda. Todo ello adquiere una mayor relevancia si cabe en los ayuntamientos, donde la estructura de los mismos, con la presencia de múltiples concejalías y departamentos, genera de por sí una lentitud comunicativa y un lenguaje muy complicado de trasladar a la ciudadanía, lo que produce un aumento de la rumorología y la insatisfacción de los usuarios de la red (Villar, 2015).

Por otro lado, las elecciones municipales de 2015 en Elche significaron un cambio radical en el equipo de gobierno ilicitano. En la ciudad formó gobierno un tripartito representado por el PSOE, Compromís e Ilicitanos por Elche (puesto ocupado desde febrero de 2016 por el Partido de Elche) tras la ruptura del primer tripartito.

En materia de comunicación, área gobernada por el partido Compromís, se decidió lanzar un plan social media con el objetivo estratégico de situar al Consistorio como un institución cercana y transparente en sus actitudes, con el apoyo de los perfiles en las redes sociales para servir de canal informativo a los ciudadanos y que así estén debidamente informados de las actividades que emanan del Ayuntamiento.

Una tendencia al alza en el sector público donde diversos entes han comenzado a profesionalizar sus comportamientos en los medios sociales y a planificar sus acciones comunicativas (Criado y Rojas, 2013). Plataformas como Twitter han demostrado su capacidad para contribuir a la autonomía informativa de los ciudadanos. Esto ha despertado el interés de los dirigentes políticos por aplicar una política de comunicación avanzada (Moya Sánchez y Damas, 2015). Como consecuencia de esta profesionalización de la comunicación en los ayuntamientos, el presente trabajo realiza un análisis del plan de comunicación en redes sociales del departamento de Comunicación del Ayuntamiento de Elche con el fin de estudiar los canales comunicativos utilizados por la organización para implementar su estrategia online.

\section{Objetivos e hipótesis}

Este trabajo pretende analizar el comportamiento de un ente público como un ayuntamiento en las redes sociales (Facebook y Twitter) y nos proponemos, basándonos en el estudio del plan de comunicación online del Ayuntamiento de Elche, los siguientes objetivos:

1. Determinar cuál es el comportamiento actual del Ayuntamiento en sus perfiles en redes sociales. 
2. Determinar el potencial de influencia del perfil en las redes sociales.

3. Analizar de qué manera el plan de comunicación online ha contribuido a generar una actitud positiva por parte de los seguidores.

4. Averiguar qué rendimiento ofrece el perfil en las redes sociales.

5. Analizar el nivel de interacción entre la cuenta y los usuarios de la red.

Por tanto, a partir de estos objetivos las hipótesis que se han tratado de verificar o refutar con la presente investigación son:

- $\quad 1^{a}$ Hipótesis: si el Ayuntamiento de Elche ha elaborado un plan de comunicación en redes sociales que le permite aprovechar los canales comunicativos de las redes sociales en su estrategia comunicativa online, situarse como una institución cercana a la ciudadanía y que le sirve de canal informativo que ayuda a resolver las dudas de los usuarios de la red, generando al mismo tiempo interacción.

- $\quad 2^{a}$ Hipótesis: si el comportamiento comunicativo en redes sociales le permite conectar con sus seguidores y generar una actitud positiva en ellos.

- $\quad 3^{a}$ Hipótesis: si las redes sociales se han convertido en un espacio social donde se comparten ideas y opiniones que han contribuido a la formación de una sensación de comunidad con usuarios activos.

\section{Marco teórico}

Las instituciones públicas han empezado en los últimos años a hacer uso de las redes sociales como el mejor canal donde transmitir al público las actividades que emanan de los distintos departamentos y concejalías que conforman, por ejemplo, un ente público como un ayuntamiento. Así lo expresa Grande (2016), quien señala que las administraciones públicas se encuentran obligadas en las últimas décadas a implementar mejoras en su funcionamiento con el fin de ofrecer un mejor servicio a los ciudadanos en el entorno digital y generar, de esta manera, un valor público.

En este sentido, los consistorios han considerado que las redes sociales suponen una de las mejores vías por las que trasladar con una mayor eficacia y rapidez cualquier contenido informativo de índole municipal. En el caso de Elche, existen 100.000 usuarios potenciales en Facebook, aproximadamente un $40 \%$ de la población total del municipio ilicitano, que cuenta, según la última estimación del año 2014 del Instituto Nacional de Estadística (INE), con un total de 228.647 habitantes (Villar, 2015).

\subsection{Las redes sociales en el ámbito de la administración pública}

En un estudio llevado a cabo por Zumba (2015), este autor presenta un análisis sobre los perfiles sociales en Facebook y Twitter de tres instituciones públicas del cantón Cuenca, en Ecuador, que usan estas herramientas como estrategia de comunicación. La investigación determina la importancia de estas redes sociales en el ámbito público como mecanismo de participación, colaboración y transparencia. A través de un análisis descriptivo de los mensajes compartidos en dichas cuentas mencionadas, el investigador averiguó la forma, los contenidos publicados y la frecuencia con la que las instituciones lo hacían. Sobre los contenidos publicados, destaca los temas institucionales, de participación y la política como predominantes y señala una presencia mínima de lo político-partidario. Si por algo se caracterizan las redes sociales es por la bidireccionalidad, ya que el emisor y el receptor pueden intercambiar mensajes y enriquecer así 
el acto comunicativo generando el conocido engagement., aunque según este autor las cuentas de las instituciones hablan mucho pero escuchan poco" (Zumba, 2015, p. 134).

En lo relativo a los mensajes vertidos en las cuentas institucionales de la administración pública, el estudio muestra que los entes públicos emiten mensajes que en la mayoría de los casos no resultan de interés para los ciudadanos, ya que van dirigidos con frecuencia a influir en la agenda de los medios, publicitar eventos o como herramienta para posicionar a la institución (Zumba, 2015, p. 134).

Por su parte, Criado Grande y Rojas (2013, p. 7) sitúan a las redes sociales como una oportunidad para las administraciones públicas para innovar y mejorar internamente, así como para profundizar en las relaciones con los ciudadanos. Estos investigadores también consideran esencial que la administración pública adopte un comportamiento activo en redes sociales para escuchar las opiniones de los usuarios con el fin de atender las peticiones de los ciudadanos y mejorar así las políticas públicas y sostienen que las redes sociales suponen una gran oportunidad para que los ciudadanos ganen presencia en el sector público, en el sentido de que su colaboración y participación en la red pueden resultar esenciales en la mejora de los servicios públicos y las políticas de los gobiernos en cuestión. (Criado Grande y Rojas, 2013, p. 29).

A modo de ejemplo, en una investigación que se llevó a cabo por Estebaranz y Ramilo (2013) se cuestionaba, en primer lugar, si las instituciones locales vascas tenían presencia en las redes sociales y en caso afirmativo, si existía estrategia de comunicación en ellas por parte de dichos órganos locales. El trabajo reflejaba que las instituciones vascas realizaban una comunicación unidireccional en redes sociales donde no existía interacción alguna con los usuarios.

Estebaranz y Ramilo (2013, p. 97) señalan que los gobiernos y administraciones públicas locales vascas no consiguen generar engagement con los usuarios y originar sentimiento de pertenencia y participación. De igual manera, Pereyra Caramé (2012) critica la presencia pasiva de los consistorios en las redes sociales, que no provoca la opinión o reacción del ciudadano en un entorno perfecto para participar en la gestión gubernamental de forma ágil, por lo que apelan a que se conviertan en una verdadera herramienta de participación para el ciudadano.

Por otro lado, Genovés (2013, p. 10) considera que las características de las redes sociales (versatilidad, agilidad, facilidad de uso...), convierten a estas herramientas en la mejor manera para comunicar y esto hace de las redes sociales un canal crucial en cualquier estrategia de comunicación en las instituciones públicas. Es importante señalar que la presencia activa de los ayuntamientos en las redes sociales permite que estos conozcan las demandas de los ciudadanos y sus opiniones acerca de las administraciones. Por esta razón, Merodio (2010, p. 60) afirma que una de las principales claves del éxito es la empatía, es decir, saber ponernos en el lugar de nuestros clientes y pensar como ellos, y algo que puede ayudar mucho es conocer directamente su opinión.

El trabajo de Vañó (2015, p. 98) señala la importancia de aportar una presencia oficial a las administraciones públicas con unos objetivos claros como la comunicación bidireccional, homogénea y coherente, que apoyen los criterios de transparencia de la institución en cuestión. Por esta razón, la investigadora defiende la importancia de sacar el máximo rendimiento a un perfil social a través de la medición de resultados, que no se basen únicamente en datos 
de seguidores sino de alcance e interacción. Y es que la implementación de herramientas de monitoreo es uno de los desafíos de estas administraciones (Campillo y Martínez, 2017, p. 513). Otros autores también señalan que la cantidad de seguidores en las redes sociales no es un parámetro que sirva para determinar el éxito o el fracaso de la gestión comunicativa de una organización en las redes sociales. La existencia de un gran número de seguidores en Facebook no asegura automáticamente una audiencia comprometida y enganchada (Haro de Rosario, et. al, p. 235) y señalan que sería útil aplicar políticas para promover la interacción entre el gobierno y la sociedad, por medio de encuestas, consultas y debates.

Otros expertos como Vañó (2015, p. 112) añaden que la comunicación en las redes sociales es un proceso que requiere de tiempo y de una planificación a largo plazo para conseguir con vocación de servicio. El autor destaca que existe cierto peligro de que la comunicación política sustituya a la institucional y señalan la necesidad de que sea el ente el que tenga visibilidad en los perfiles de las redes sociales corporativas en lugar de los partidos políticos.

Para finalizar, los expertos señalan la necesidad de que los ciudadanos ganen presencia en la vida pública con su participación activa en la toma de decisiones. Al respecto, Sande (2016) insiste en la necesidad de huir de los intereses de los propios partidos y que el ciudadano se convierta en el eje de la comunicación pública e institucional. Precisamente, Fenoll y Cano-Oron (2017) destacan que en la actualidad se está produciendo unas sustanciales diferencias de participación en las redes sociales entre los seguidores de partidos tradicionales y las nuevas formaciones políticas, que trasciende a estas plataformas la polarización de sus comportamientos en la vida política.

\section{Metodología}

La metodología usada para la presente investigación sobres los perfiles corporativos en redes sociales del Ayuntamiento de Elche es empírico-analítica, y para ello se ha utilizado la técnica del análisis de contenido. Noguera Vivo (2010, p. 182), explica la capacidad organizativa y concluyente que presenta dicho método para obtener resultados satisfactorios del análisis de la actividad de los distintos perfiles de Facebook en relación a parámetros como la conexión, la participación, los contenidos y la dinamización. Por su parte, Castelló et al. (2014, p. 35) muestran que el análisis de contenido es una herramienta idónea para estudiar y analizar el comportamiento y la actividad de las cuentas sociales en Twitter, así como la interacción de los usuarios con las mismas.

Con el fin de establecer una serie de conclusiones que permitan verificar o rechazar las hipótesis planteadas tras la observación de diversos estudios similares publicados por otros autores, se ha realizado una investigación cuantitativa y cualitativa de las cuentas oficiales del Ayuntamiento de Elche en Facebook y Twitter. A nivel cuantitativo se han medido variables como el número de likes, seguidores, comentarios..., con el fin de cuantificar el total mientras que en la parte cualitativa se ha puesto el foco en el análisis de aspectos relacionados con los tipos de comentarios y respuestas, entre otros.

Para el primer caso se ha analizado el perfil de Facebook@ayuntamientoelche, mientras que el segundo se ha centrado en la cuenta de Twitter@ajuntamentelx. Este trabajo de análisis de datos ha tenido una duración de dos semanas, del 17 de abril al 30 de abril de 2017. La elección de este periodo de tiempo responde a diversas controversias en la ciudad, como fueron 
la fumigación para prevenir las plagas de mosquito, especialmente el peligros mosquito tigre, el requisito del valenciano como acceso a una plaza pública y los criterios de acceso a las escuelas municipales infantiles.

Este análisis de contenido ha requerido de una continua recogida de información diaria que se ha ido clasificando en las distintas tablas elaboradas para medir las variables propuestas en los objetivos de la presente investigación.

Para el monitoreo de los datos, tanto en Facebook como Twitter, se ha hecho uso de la herramienta Fanpage Karma. Este sistema de estadísticas permite medir aspectos fundamentales para los objetivos propuestos como son la presencia y actividad de la cuenta, el nivel de interacción que se genera entre el perfil y los usuarios así como las reacciones de estos últimos a las publicaciones de los dos perfiles objetos de estudio.

\subsection{Variables analizadas en Twitter}

En primer lugar, para la descripción de la muestra del perfil@ajuntamentelx en Twitter se dividen los datos en cuatro fases distintas de estudio. La presencia del perfil conforma la primera parte de la recogida de estadísticas. En este apartado se han analizado aspectos como el número de seguidores y seguidos por la cuenta y el potencial de influencia del perfil. El segundo bloque de análisis se centra en la actividad del perfil durante las dos semanas de estudio, así se han recogido datos en relación al número de tweets publicados en total y por día, número de hashtags usados y el índice de rendimiento de la cuenta. En lo que hace referencia al tercer bloque, los usuarios han sido el objeto de estudio ya que se han cuantificado sus reacciones en cuanto al número de retweets, "me gusta" y respuestas realizadas sobre la cuenta, así como el número de "me gusta" y retweets recogidos de media por cada tweet publicado.

Por último, la interacción ha sido estudiada de acuerdo con parámetros como la interacción de los tweets, el porcentaje de conversaciones generadas durante el periodo de estudio o el índice de compromiso de los usuarios con la cuenta. Por otra parte, con un componente más cualitativo, se han dividido las respuestas de los usuarios en cuatro tipos (positivas, negativas y peticiones de información) con el fin de observar la tipología predominante.

En la siguiente tabla se resumen las variables de estudio para el análisis de la cuenta@ ajuntamentelx en Twitter (García Mogedas, 2015).

Cuadro 1. Resumen de las variables de estudio en el perfil de Twitter. Fuente: Elaboración propia.

\begin{tabular}{|c|c|c|c|}
\hline PRESENCIA & ACTIVIDAD & REACCIONES & INTERACCIÓN \\
\hline $\begin{array}{ll}\text { - } & \mathrm{N}^{\circ} \text { Seguidores } \\
\text { - } & \mathrm{N}^{\circ} \text { Seguidos } \\
\text { - } & \text { Ratio seguidores } \\
& \text { / seguidos }\end{array}$ & $\begin{array}{ll} & \mathrm{N}^{\circ} \text { Tweets } \\
& \text { totales / día } \\
\text { - } & \mathrm{N}^{\circ} \text { Hashtags } \\
\text { - } & \text { Índice de } \\
& \text { rendimiento }\end{array}$ & $\begin{array}{ll}\text { - } & \mathrm{N}^{\circ} \text { Retweets } \\
\text { totales / tweet } \\
\text { - } & \mathrm{N}^{\circ} \text { Me gusta } \\
\text { totales / tweet } \\
\text { - } \mathrm{N}^{\circ} \text { Respuestas } \\
\text { totales }\end{array}$ & $\begin{array}{ll}\text { - } & \text { Interacción tweets } \\
\text { - } & \text { \% Conversación } \\
\text { - } & \text { Indice de compromiso } \\
& \text { Tipología de respuesta } \\
& \text { (positiva, negativa, } \\
\text { neutra, petición de } \\
\text { info.) }\end{array}$ \\
\hline
\end{tabular}




\subsection{Variables analizadas en Facebook}

En lo que al análisis de la cuenta de Facebook se refiere, se ha utilizado el mismo procedimiento que en Twitter para la extracción de datos, sin embargo las tablas de análisis son diferentes ya que cada red social cuenta con unas particularidades, por lo que las variables a medir serán distintas en algunos casos.

A pesar de las diferencias mencionadas, la metodología es similar y el análisis de contenido se centra de igual manera en cuatro grandes bloques: presencia del perfil, actividad generada por la cuenta, reacciones de los usuarios e interacción.

En el primer gran bloque se han mostrado los datos referentes al número de fans que tiene la página en Facebook. Por su parte de la actividad de la cuenta se ha extraído información relativa a la muestra de publicaciones recogidas durante el periodo de análisis así como el índice de rendimiento de la página. El tercer bloque hace referencia a las reacciones de los usuarios a las publicaciones, una manera de medir la actitud y el comportamiento de los usuarios través del número de reacciones (número de "me gusta", número de "me enfada"...), el número de compartidos y el total de comentarios de los usuarios registrados en la cuenta.

Por último, se ha hecho referencia a la interacción. En este último punto de estudio se han analizado variables como la interacción de las publicaciones, el índice de compromiso por parte de los fans hacia la página, las conversaciones entre el emisor y los receptores de la información y la tipología de comentarios (positivos, negativos, peticiones de información...).

En la siguiente tabla se resumen las variables de estudio para el análisis de la cuenta @ ayuntamientoelche en Facebook (García Mogedas, 2015).

Cuadro 2. Resumen de las variables de estudio en el perfil de Facebook. Fuente: Elaboración propia.

\begin{tabular}{|c|c|c|c|}
\hline PRESENCIA & ACTIVIDAD & REACCIONES & INTERACCIÓN \\
\hline - $\quad \mathrm{N}^{\mathrm{o}}$ Fans & $\begin{array}{ll}\text { - } & \mathrm{N}^{\mathrm{o}} \text { Posts } \\
& \text { totales / día } \\
\text { - } & \text { Índice de } \\
& \text { rendimiento } \\
\text { de la página }\end{array}$ & $\begin{array}{ll}\text { - } & \mathrm{N}^{0} \text { Reacciones } \\
\text { - } & \mathrm{N}^{0} \text { Comentarios } \\
\text { - } & \mathrm{N}^{\mathrm{o}} \text { Compartidos } \\
& \mathrm{N}^{\mathrm{o}} \text { Total Reacciones, } \\
& \text { Comentarios y Com- } \\
& \text { partidos }\end{array}$ & $\begin{array}{ll}\text { - } & \text { Interacción publicaciones } \\
\text { - } & \text { / post } \\
\text { - } & \text { Índice de compromiso } \\
\text { - } \quad \text { Tipología de comentarios } \\
\text { (positivos, negativos, pe- } \\
\text { ticiones de información, } \\
\text { otros...) }\end{array}$ \\
\hline
\end{tabular}

\section{Resultados}

Una vez han sido monitorizados los datos referentes a las cuentas corporativas del Ayuntamiento de Elche en Twitter y Facebook, se procede al análisis de los resultados extraídos a lo largo del periodo de estudio con el fin de verificar o refutar las hipótesis planteadas al inicio de la presente investigación. 


\subsection{Resultados del análisis en Twitter}

Como se ha mencionado en el apartado de la metodología, el estudio se centra en el análisis de cuatro aspectos fundamentales como la presencia del perfil en Twitter, la actividad del emisor en la red social, las reacciones de los usuarios y la interacción entre ambas partes del proceso de comunicación como son el Ayuntamiento y sus seguidores.

Cuadro 3. Presencia del perfil en Twitter durante el periodo de estudio. Fuente: Elaboración propia.

\begin{tabular}{|c|c|c|}
\hline Página & $\mathbf{N}^{\mathbf{0}}$ Seguidores & $\mathbf{N}^{\mathbf{0}}$ Seguidos \\
\hline @ajuntamentelx & 15.505 & 545 \\
\hline
\end{tabular}

Según podemos observar en el cuadro 3, el Ayuntamiento de Elche, al inicio de la presente investigación, presentaba un total de 15.505 seguidores en la red social de Twitter, además seguía a un número de 545 perfiles. Sin embargo, el hecho de contar con un gran número de seguidores no tiene por qué ser una garantía de éxito (Haro de Rosario et al., 2016, p. 235).

Cuadro 4. Ratio de seguidores y seguidos en Twitter. Fuente: Elaboración propia.

\begin{tabular}{|c|c|c|c|}
\hline Página & $\mathbf{N}^{\text {o }}$ Seguidores & $\mathbf{N}^{\mathbf{0}}$ Seguidos & $\begin{array}{c}\text { Ratio seguidores / } \\
\text { seguidos }\end{array}$ \\
\hline @ajuntamentelx & 15.147 & 543 & 27,89 \\
\hline
\end{tabular}

El cuadro 4 muestra el cálculo del potencial de influencia de la cuenta en Twitter, para ello se ha dividido entre el número de seguidores y seguidos. El resultado muestra una gran relevancia y autoridad de la cuenta, ya que por cada perfil que@ajuntamentelx sigue, recibe una media de 27,89 seguidores.

Cuadro 5. Actividad de la cuenta en Twitter durante el periodo de estudio. Fuente: Elaboración propia.

\begin{tabular}{|c|c|c|c|c|}
\hline Página & $\mathbf{N}^{\mathbf{o}}$ Tweets & $\begin{array}{c}\mathbf{N}^{\mathbf{0}} \\
\text { Tweets / día }\end{array}$ & $\begin{array}{c}\mathbf{N}^{\mathbf{0}} \\
\text { Hashtags }\end{array}$ & $\begin{array}{c}\text { Índice de } \\
\text { rendimiento }\end{array}$ \\
\hline @ajuntamentelx & 63 & 4,5 & 1 & $34 \%$ \\
\hline
\end{tabular}

La actividad de la cuenta deja una muestra total de 63 tweets publicados durante el periodo de análisis. Por otra parte, la herramienta Fanpage Karma muestra la frecuencia de publicación del perfil y la sitúa en 4,5 tweets por día en las dos semanas observadas. En esta actividad se aprecia la ausencia de comunicación con el usuario y esto queda reflejado en el número de hashtags utilizados a lo largo de la investigación. 
Ajuntament d'Elx @ajuntamentelx-25 abr.

Se da cuenta de asuntos del área económica. \#EIxPle

4 47

Ajuntament d'Elx@ajuntamentelx.25 abr.

Comença el Ple de l'Ajuntament d'Elx.

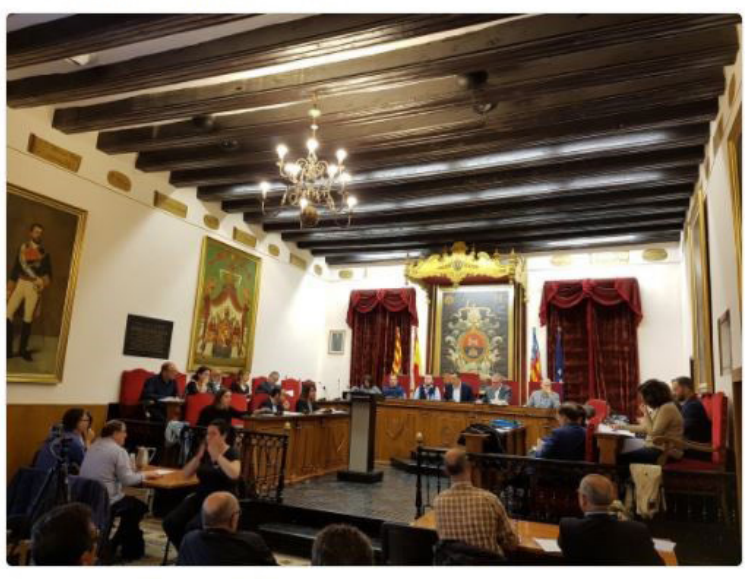

4 477 난

Imagen 1. Hashtag utilizado durante el pleno municipal celebrado en abril. Fuente: Twitter

La imagen 1 corresponde a un tweet publicado durante la celebración del pleno mensual en el Ayuntamiento de Elche. Como se puede observar, para ese día el Community Manager de la institución pública utilizó el hashtag \#ElxPle, pero esta etiqueta no registró ninguna respuesta por parte de los usuarios a pesar de que el pleno fue retransmitido por streaming a través del canal de Youtube del ente ilicitano.

Las etiquetas o hashtags son una buena manera de fomentar la escucha activa de los usuarios y contribuir al seguimiento del acto, tal y como sostienen Estebaranz y Ramilo (2013, p. 97), quienes defienden las posibilidades de interacción que proporciona el uso de estas etiquetas.

En la red social Twitter las reacciones de los usuarios se han medido a través de tres variables: los retweets, los likes y las respuestas o comentarios sobre cualquier tweet publicado por la cuenta.

Cuadro 6. Reacción de los usuarios a las publicaciones de la cuenta@ajuntamentelx durante el periodo de estudio. Fuente: Elaboración propia.

\begin{tabular}{|c|c|c|c|}
\hline Página & $\mathbf{N}^{\mathbf{o}}$ Retweets & $\mathbf{N}^{\mathbf{o}}$ Me gusta & $\mathbf{N}^{\mathbf{o}}$ Respuestas \\
\hline @ajuntamentelx & 90 & 102 & 4 \\
\hline
\end{tabular}

A El siguiente cuadro, el número 6, muestra la reacción de los usuarios a la actividad generada por la cuenta@ajuntamentelx. En las dos semanas de estudio, se contabilizaron un total de 63 tweets, y estos a su vez dieron lugar a 90 retweets, 102 likes y 4 respuestas por parte de los seguidores. 
Cuadro 7. Número de reacciones de los usuarios por tweet durante el periodo de estudio. Fuente: Elaboración propia.

\begin{tabular}{|c|c|c|}
\hline Página & $\begin{array}{c}\mathbf{N}^{\mathbf{o}} \text { Me gusta / } \\
\text { tweets }\end{array}$ & $\mathbf{N}^{\mathbf{o}}$ Retweets / tweet \\
\hline @ajuntamentelx & 1,6 & 1,4 \\
\hline
\end{tabular}

El número de reacciones de los usuarios por tweet muestra un número de likes y retweets muy bajo. La herramienta Fanpage Karma extrae un 1,6 de likes por cada tweet publicado y 1,4 retweets por cada tweet emitido. Esta información indica una actitud pasiva de los usuarios ante la actividad del Ayuntamiento en Twitter (ver cuadro 7).

Cuadro 8. Interacción entre la cuenta y los usuarios durante el periodo de estudio. Fuente: Elaboración propia.

\begin{tabular}{|c|c|c|c|}
\hline Página & $\begin{array}{c}\text { Interacción de } \\
\text { tweets }\end{array}$ & Conversaciones & $\begin{array}{c}\text { Índice de com- } \\
\text { promiso }\end{array}$ \\
\hline @ajuntamentelx & $0,020 \%$ & $0 \%$ & $0,091 \%$ \\
\hline
\end{tabular}

La interacción es el último bloque que se ha analizado en la red social Twitter y de él se pueden extraer datos proporcionados por Fanpage Karma referentes a la interacción de los tweets, el porcentaje de conversación generado durante el periodo de estudio y el índice de compromiso de los usuarios con el perfil del Consistorio.

El cuadro 8 muestra un porcentaje del 0,020\% de interacción en la muestra de 63 tweets seleccionada para la presente investigación y un $0 \%$ de conversaciones, es decir, ninguno de los 4 respuestas de los usuarios obtenidas en este periodo de estudio han recibido contestación por parte del perfil@ajuntamentelx. Todo ello deriva en un compromiso escaso por parte de los usuarios y así lo señala el índice de compromiso de Fanpage Karma que revela un 0,091\%.

Cuadro 9. Tipología de respuestas de los usuarios a los tweets de la cuenta@ajuntamentelx durante el periodo de estudio. Fuente: Elaboración propia.

\begin{tabular}{|c|c|c|c|c|}
\hline Página & $\begin{array}{c}\mathbf{N}^{\mathbf{o}} \text { Respuestas } \\
\text { positivas }\end{array}$ & $\begin{array}{c}\mathbf{N}^{\mathbf{o}} \text { Respuestas } \\
\text { negativas }\end{array}$ & $\begin{array}{c}\mathbf{N}^{\mathbf{o}} \text { Peticiones } \\
\text { de info. }\end{array}$ & $\begin{array}{c}\mathbf{N}^{\mathbf{o}} \text { Total } \\
\text { respuestas }\end{array}$ \\
\hline @ajuntamentelx & 1 & 1 & 2 & 4 \\
\hline
\end{tabular}

Tal y como se ha mencionado anteriormente, el perfil @ajuntamentelx tan sólo ha registrado 4 respuestas de usuarios durante las dos semanas de estudio. Este dato muestra una implicación mínima de los seguidores con la cuenta a la hora de conversar con el emisor. Sin embargo, este hecho se puede explicar debido a la actitud unidireccional que el Ayuntamiento tiene en Twitter.

El cuadro 9 muestra la tipología de comentarios realizados por los usuarios de la red, y en él, 
podemos observar que un 50\% de las respuestas emitidas por los seguidores son peticiones de información en relación a noticias publicadas desde el Consistorio.

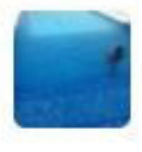

bambola@aprender_b.18 abr.

En respuesta a @ajuntamentelx

Cuando se va a fumigar en las bayas???es imposible con tanto mosquito

Imagen 2. Respuesta de un usuario a un tweet publicado el 18 de abril. Fuente: Twitter.

En la imagen 2 podemos ver la petición de información por parte de un usuario sobre una tema como la fumigación de una partida de Elche, las Bayas, debido a la proliferación de mosquitos en la zona. El perfil, ante dicha cuestión, no emite ninguna contestación y el seguidor observa que su duda no ha sido resuelta. Esta situación genera descontento en el público de la red, ya que recibe la percepción de un perfil que publica contenidos pero que no replica contestación alguna.

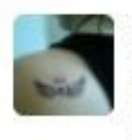

ceci @_sesy_sesy·-18 abr.

En respuesta a @ajuntamentelx

Eso está bien, porque yo soy alérgica!!!

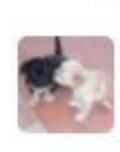

JUAN CARLOS@JUANCARLOSPQ82-18 abr.

En respuesta a @ajuntamentelx

Seguro, fumigaciones... Tratamientos gaseosos.... Me da q no. Básicamente

seguir las recomendaciones y si t pican $t$ rascas

Imagen 3. Respuestas de los usuarios a un tweet del 18 de abril. Fuente: Twitter.

En este caso, la imagen 3 se corresponde con dos respuestas que los usuarios ofrecieron a un tweet publicado el día 18 de abril. En él, se registran dos comentarios de usuarios que expresan sus opiniones a través de botón de respuesta. El primero de ellos es positivo y muestra su alegría ante la noticia de que el Ayuntamiento intensificaría la lucha contra el mosquito tigre, mientras que el segundo es negativo y cuestiona las medidas llevadas a cabo por el Consistorio para acabar con las picaduras del insecto. Ninguna de las dos respuestas reciben reacción por parte del emisor de la información, ya sea en forma de like, retweet o respuesta.

JUAN CARLOS @JUANCARLOSPQ82-18 abr.

En respuesta a @ajuntamentelx

Seguro, fumigaciones.... Tratamientos gaseosos.... Me da q no. Básicamente seguir las recomendaciones y si t pican $t$ rascas

Imagen 4. Reacción de un usuario a un tweet publicado el 19 de abril. Fuente: Twitter. 
En la imagen 4 se puede ver de nuevo una falta de comunicación entre el perfil y un seguidor que solicita información sobre la edad requerida para utilizar el servicio de autobús gratuito para estudiantes.

Resulta curioso que el usuario que pide información también lo hace en otra ocasión tal y como se observa en la imagen 3. Los usuarios necesitan sentirse realizados y probablemente este seguidor con el paso del tiempo deje de utilizar el canal de Twitter para informarse ante la actitud unidireccionaldel perfil@ajuntamentelx.

\subsection{Resultados del análisis en Facebook}

Una vez han sido desgranados todos los datos vertidos de la actividad proporcionada por la cuenta en Twitter durante la fase de estudio, se procede a la descripción de la gestión comunicativa que el ente público realiza en su página de Facebook.

Cuadro 10. Presencia del perfil en Facebook durante el periodo de estudio. Fuente: Elaboración Propia.

\begin{tabular}{|c|c|}
\hline Página & $\begin{array}{c}\text { Número de } \\
\text { fans }\end{array}$ \\
\hline $\begin{array}{c}\text { Ayuntamiento de } \\
\text { Elche - Ajuntament } \\
\text { d'Elx }\end{array}$ & 13.421 \\
\hline
\end{tabular}

La presencia de la página Ayuntamiento de Elche - Ajuntament d'Elx en Facebook refleja una cifra de 13.421 fans en el inicio de la investigación y así lo demuestra el cuadro 10 el cual indica la presencia de la página en la red social.

Cuadro 11. Actividad de la cuenta en Facebook durante el periodo de estudio. Fuente: Elaboración Propia.

\begin{tabular}{|c|c|c|c|}
\hline Página & No Publicaciones & $\begin{array}{c}\mathbf{N}^{\mathbf{o}} \text { Publicaciones } \\
\text { / día }\end{array}$ & $\begin{array}{c}\text { Índice de } \\
\text { Rendimiento } \\
\text { de la Página }\end{array}$ \\
\hline $\begin{array}{c}\text { Ayuntamiento de Elche } \\
\text { - Ajuntament d'Elx }\end{array}$ & 51 & 3,6 & $43,0 \%$ \\
\hline
\end{tabular}

En lo que hace referencia a la actividad generada por la página durante el periodo de estudio, el cuadro 11 da como resultado una muestra de 51 publicaciones, que hacen una media de 3,6 publicaciones por cada día analizado. Además la herramienta Fanpage Karma ofrece datos referentes al rendimiento del perfil y este índice muestra un $43 \%$ de rendimiento de la página. 
Cuadro 12. Reacción de los usuarios a las publicaciones de la cuenta durante el periodo de estudio. Fuente: Elaboración Propia.

\begin{tabular}{|c|c|c|c|c|c|c|c|c|}
\hline Página & $\begin{array}{c}\mathbf{N}^{\mathbf{0}} \mathbf{M e} \\
\text { gusta }\end{array}$ & $\begin{array}{c}\mathbf{N}^{\mathbf{0}} \mathbf{M e} \\
\text { encanta }\end{array}$ & $\begin{array}{c}\mathbf{N}^{\mathbf{0}} \mathbf{M e} \\
\text { asombra }\end{array}$ & $\begin{array}{c}\mathbf{N}^{\mathbf{0}} \mathbf{M e} \\
\text { divierte }\end{array}$ & $\begin{array}{c}\mathbf{N}^{\mathbf{o}} \mathbf{M e} \\
\text { entris- } \\
\text { tece }\end{array}$ & $\begin{array}{c}\mathbf{N}^{\mathbf{o}} \mathbf{M e} \\
\text { enor- } \\
\text { gullece }\end{array}$ & $\begin{array}{c}\mathbf{N}^{\mathbf{2}} \\
\mathbf{M e} \\
\text { enfa- } \\
\text { da }\end{array}$ & $\begin{array}{c}\mathbf{N}^{\mathbf{T}} \\
\text { Total } \\
\text { reac- } \\
\text { cio- } \\
\text { nes }\end{array}$ \\
\hline $\begin{array}{c}\text { Ayuntamiento } \\
\text { de Elche- } \\
\text { Ajuntament } \\
\text { d'Elx }\end{array}$ & 904 & 94 & 0 & 1 & 1 & 0 & 4 & 1.005 \\
\hline
\end{tabular}

El cuadro 12, por su parte, es la primera de las tablas que mide las variables relacionadas con las reacciones de los usuarios. Durante la fase de recogida de datos se obtiene un total de 1.005 reacciones de las 51 publicaciones realizadas por el perfil del Consistorio. Estas reacciones se dividen en 904 likes; 94 "me encanta"; 0 "me asombra"; 1 "me divierte"; 1 "me entristece"; 0 "me enorgullece" y 4 "me enfada".

Cuadro 13. Número de reacciones de los usuarios por publicación durante el periodo de estudio. Fuente: Elaboración Propia.

\begin{tabular}{|c|c|c|c|c|c|c|c|c|}
\hline Página & $\begin{array}{l}\mathrm{N}^{\circ} \mathrm{Me} \\
\text { gusta } \\
\text { / post }\end{array}$ & $\begin{array}{c}\mathbf{N}^{\circ} \mathbf{M e} \\
\text { encanta } \\
\text { / post }\end{array}$ & $\begin{array}{c}\mathrm{N}^{\circ} \mathrm{Me} \\
\text { asombra } \\
\text { / post }\end{array}$ & $\begin{array}{c}\mathbf{N}^{o} \mathrm{Me} \\
\text { divierte } \\
\text { / post }\end{array}$ & $\begin{array}{c}\mathrm{N}^{\circ} \mathrm{Me} \\
\text { entris- } \\
\text { tece / } \\
\text { post }\end{array}$ & $\begin{array}{c}\mathrm{N}^{\circ} \mathrm{Me} \\
\text { enor- } \\
\text { gullece } \\
\text { / post }\end{array}$ & $\begin{array}{l}\mathrm{N}^{\mathbf{}} \\
\mathrm{Me} \\
\text { enfa- } \\
\text { da / } \\
\text { post }\end{array}$ & $\begin{array}{c}\mathrm{N}^{\circ} \\
\text { Total } \\
\text { reac- } \\
\text { cio- } \\
\text { nes } / \\
\text { post }\end{array}$ \\
\hline $\begin{array}{c}\text { Ayuntamiento } \\
\text { de Elche - } \\
\text { Ajuntament } \\
\text { d'Elx }\end{array}$ & 18 & 1,8 & 0 & 0,02 & 0,02 & 0 & 0,08 & 20 \\
\hline
\end{tabular}

Por otro lado, el cuadro 13 mide el número de reacciones de los usuarios por publicación durante el periodo de estudio. De esta manera, por cada publicación se registra un volumen de 18 "me gusta"; 1,8 "me encanta"; 0 "me asombra"; 0,02 "me divierte"; 0,02 "me entristece"; 0 "me enorgullece"; 0,08 "me enfada". En resumen, el número total de reacciones por cada post se sitúa en 20.

Cuadro 14. Número total de reacciones, comentarios y compartidos por los usuarios durante el periodo de estudio. Fuente: Elaboración Propia.

\begin{tabular}{|c|c|c|c|c|}
\hline Página & $\begin{array}{c}\mathbf{N}^{\mathbf{o}} \text { de } \\
\text { Reacciones }\end{array}$ & $\begin{array}{c}\mathbf{N}^{\mathbf{o}} \text { de } \\
\text { Comentarios }\end{array}$ & $\begin{array}{c}\mathbf{N}^{\mathbf{o}} \text { de } \\
\text { Compartidos }\end{array}$ & $\mathbf{N}^{\mathbf{o}}$ Total \\
\hline $\begin{array}{c}\text { Ayuntamiento de Elche } \\
\text { - Ajuntament d'Elx }\end{array}$ & 1.005 & 141 & 1.151 & 2.297 \\
\hline
\end{tabular}


Sin embargo, las reacciones de los usuarios también se pueden recoger a través de otras variables como los comentarios o compartidos. De esta manera, se extrae un número de 141 comentarios y 1.151 compartidos. Estas dos cifras sumadas a las 1.005 reacciones hacen un total de 2.297 en las 51 publicaciones analizadas durante la presente investigación, tal y como se observa en el cuadro 14.

Cuadro 15. Interacción entre la cuenta y los usuarios durante el periodo de estudio. Fuente: Elaboración Propia.

\begin{tabular}{|c|c|c|c|c|c|}
\hline Página & $\begin{array}{c}\mathbf{N}^{\mathbf{0}} \\
\text { Comen- } \\
\text { tarios }\end{array}$ & $\begin{array}{c}\text { Interacción } \\
\text { de las } \\
\text { publicacio- } \\
\text { nes }\end{array}$ & $\begin{array}{c}\text { Interacción } \\
/ \text { publica- } \\
\text { ción }\end{array}$ & $\begin{array}{c}\% \\
\text { Conver- } \\
\text { saciones }\end{array}$ & $\begin{array}{c}\text { Índice } \\
\text { de } \\
\text { compro- } \\
\text { miso }\end{array}$ \\
\hline $\begin{array}{c}\text { Ayuntamiento de } \\
\text { Elche-Ajuntament } \\
\text { d'Elx }\end{array}$ & 141 & $0,34 \%$ & $0,70 \%$ & $3,54 \%$ & $0,83 \%$ \\
\hline
\end{tabular}

A Una buena manera de estudiar la interacción entre emisor y receptores de los contenidos es analizar los comentarios que se producen en la cuenta. Esta es la vía que tiene los usuarios para expresar sus pensamientos y opiniones a la vez que el perfil del Ayuntamiento puede replicar esos comentarios con el fin de satisfacer las necesidades de los seguidores. De esta manera se enriquece el proceso comunicativo al generar un feedback que permite al emisor recoger el punto de vista del seguidor y devolver una respuesta en referencia al mensaje (García Rico, 2015).

A lo largo del periodo de estudio se han podido extraer un total de 141 comentarios de las 51 publicaciones que conforman la muestra de estudio. De estos comentarios se observa una interacción media de $0,34 \%$, un porcentaje realmente bajo si tenemos en cuenta que la página en Facebook genera un total de 2.297 reacciones, comentarios y compartidos. Así, la interacción por publicación se establece en un $0,70 \%$.

La herramienta Fanpage Karma permite fijar un porcentaje de conversaciones entre emisor y receptores. Esta variable es fundamental para evaluar el engagement y la atención que la cuenta ofrece a sus usuarios.

El índice de conversación es de un 3,54\% según la herramienta de análisis utilizada, de lo que se puede concluir que la página realiza una comunicación unidireccional y que apenas presenta interacción con los usuarios (Estebaranz y Ramilo, 2013, p. 96).

El bajo nivel de atención y servicio proporcionado por la página del Consistorio deriva en un también ínfimo índice de compromiso por parte de la audiencia, el cual desvela un porcentaje del 0,83\%, (ver cuadro 15). 
Cuadro 16. Tipología de comentarios de los usuarios durante el periodo de estudio. Fuente: Elaboración Propia.

\begin{tabular}{|c|c|c|c|c|c|}
\hline Página & $\begin{array}{c}\mathbf{N}^{\mathbf{o}} \text { Co- } \\
\text { mentarios } \\
\text { positivos }\end{array}$ & $\begin{array}{c}\mathbf{N}^{\mathbf{o}} \text { Co- } \\
\text { mentarios } \\
\text { negativos }\end{array}$ & $\begin{array}{c}\mathbf{N}^{\mathbf{o}} \text { Peticio- } \\
\text { nes de in- } \\
\text { formación }\end{array}$ & Otros & $\begin{array}{c}\mathbf{N}^{\mathbf{o}} \text { Total } \\
\text { comen- } \\
\text { tarios }\end{array}$ \\
\hline $\begin{array}{c}\text { Ayuntamiento de } \\
\text { Elche-Ajunta- } \\
\text { ment d'Elx }\end{array}$ & 17 & 15 & 8 & 101 & 141 \\
\hline
\end{tabular}

El último cuadro de la presente investigación (ver cuadro 16) se centra en los distintos tipos de comentarios que los usuarios realizan en la cuenta oficial del Ayuntamiento.

Para estudiar esta variables se establecen cuatro tipos: positivos, negativos, peticiones de información y otros, tal y como se explica en la metodología de trabajo. En total se obtiene una cifra de 141 comentarios durante el periodo de estudio, el cual deja una muestra de 51 publicaciones.

Según los comentarios extraídos y de acuerdo con la clasificación establecida en la metodología, se observa un número de 17 comentarios positivos (12,05\%), 15 negativos $(10,63 \%), 8$ peticiones de información $(5,67 \%)$ y 101 otros $(71,63 \%)$.

Dentro de esta última categoría se encuentran comentarios que tan sólo indican una mención hacia otro usuario o aquellos que no presentan contenido con valoración personal o búsqueda de información

.Los resultados muestran un equilibrio entre los comentarios positivos y los negativos y por otro lado revelan un porcentaje relativamente bajo de comentarios informativos, ya que este valor se sitúa en un 5,67\%, es decir, de 141 comentarios tan sólo 8 son informativos.

Dejando a un lado los 101 comentarios categorizados como "otros", nos hemos centrado en la interacción presente en los comentarios positivos, negativos e informativos con el fin de visualizar las relaciones que se dan entre el emisor y los receptores de la información en el proceso comunicativo para, de esta manera, obtener una información cualitativa que complemente a los valores cuantificados en el cuadro 16. 


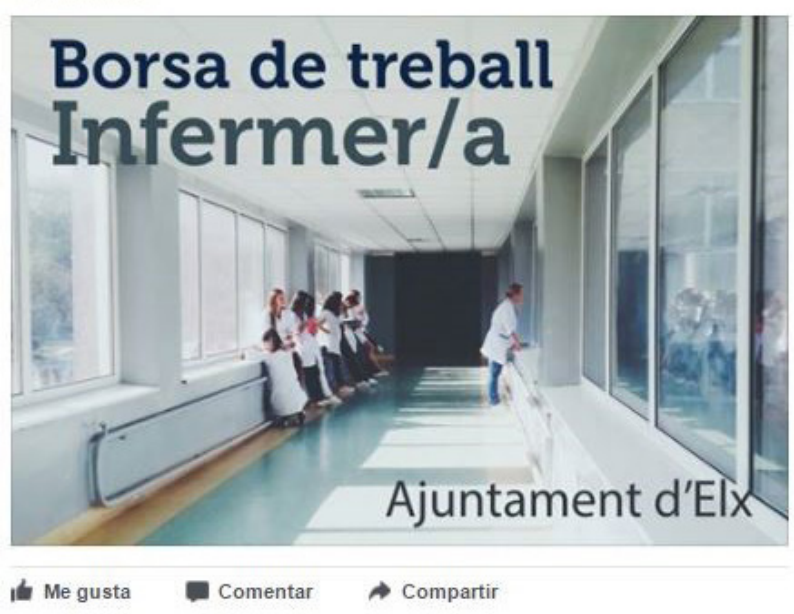

Imagen 5. Publicación de la cuenta con más actividad durante el periodo de estudio. Fuente: Facebook.

La imagen 5 muestra un post publicado por la cuenta el 19 de abril sobre la apertura de una bolsa de trabajo para enfermeros. Dicho contenido generó gran interacción y reacciones por parte de los usuarios, ya que por ejemplo, de los 141 comentarios recogidos a través de los 51 posts de la muestra, 85 de ellos se corresponden con la mencionada publicación, es decir, nada más y nada menos que un 60,28\% del total de respuestas recibidas por la página objeto de estudio. Además, el post obtuvo un total de 555 compartidos y un total de 259 reacciones (251 "me gusta", 4 "me encanta" y 4 "me enfada").

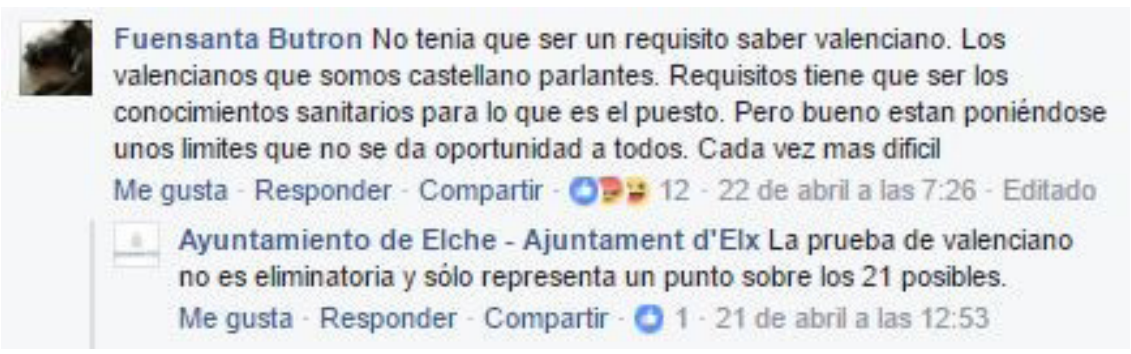

Imagen 6. Interacción entre la cuenta y un seguidor. Fuente: Facebook.

Uno de los aspectos que más polémica generó en redes sobre la apertura de la bolsa de empleo para enfermeros estuvo relacionado con el idioma valenciano, ya que fueron diversos los usuarios que mostraron su descontento con los requisitos para optar a una plaza (ver imagen 6). 
Ante este comentario el Ayuntamiento contesta al seguidor ofreciéndole datos sobre las bases como la condición no eliminatoria de la prueba en valenciano y su puntuación de 1 sobre 21 en la candidatura de acceso.

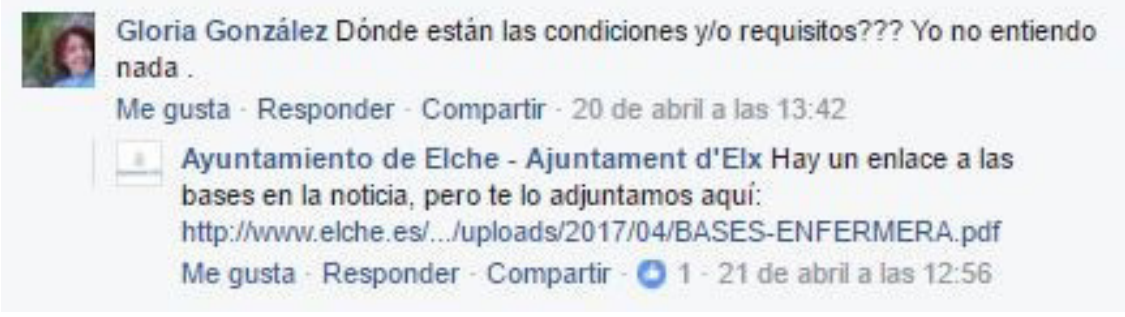

Imagen 7. Interacción entre la cuenta y un seguidor. Fuente: Facebook.

La atención personalizada sobre los usuarios es fundamental para conseguir fidelizar y generar una sensación de comunidad fuerte y de espacio abierto a la ciudadanía. La imagen 7 recoge la pregunta de un usuario sobre los requisitos de la bolsa de empleo a la que el Ayuntamiento proporciona una respuesta con un enlace externo para satisfacer la duda del solicitante.

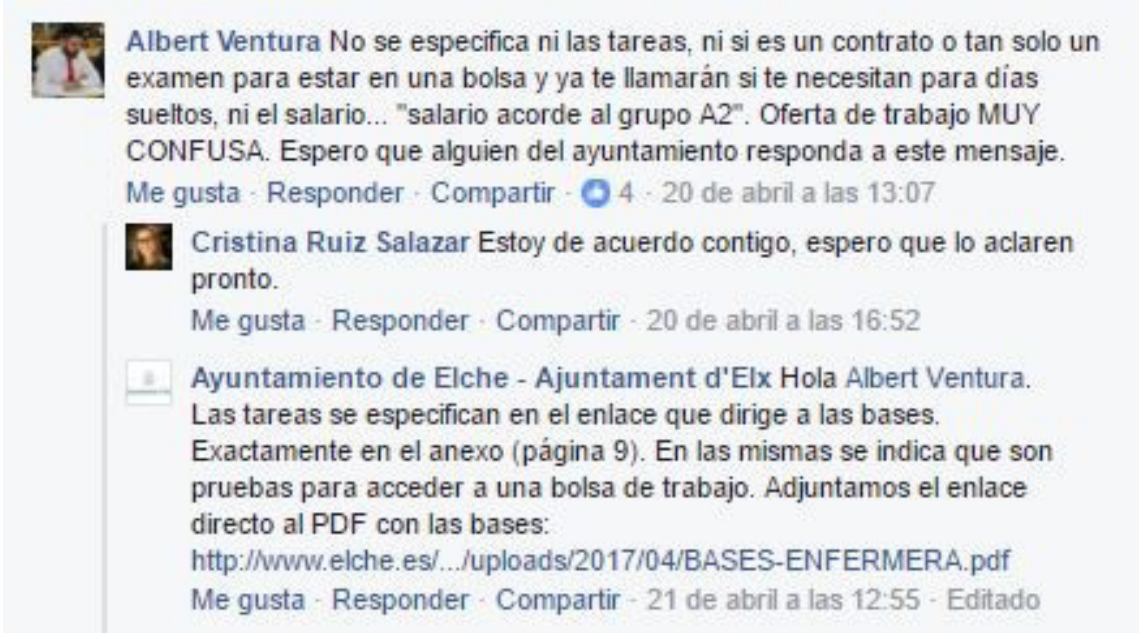

Imagen 8. Interacción entre la cuenta y un seguidor. Fuente: Facebook.

En la imagen 8 un usuario califica a la oferta de empleo como "confusa" e insta al Consistorio a aclarar ciertos aspectos de la misma. Ante este comentario la cuenta reacciona con un saludo y mención al seguidor para después ofrecer un enlace externo con toda la información. En este caso el Ayuntamiento actúa correctamente ya que amplía la información del usuario y cubre su necesidad.

Tal y como se ha podido observar de la actividad del post sobre la bolsa de empleo para enfermeros, la cuenta presenta una actitud de predisposición a ayudar a los usuarios con sus dudas sobre la candidatura facilitando más información a través de un mensaje claro y correcto. Sin embargo, ¿cómo se ha comportado la cuenta ante las dudas de los usuarios en otras publicaciones con temática diferente? 


\section{Ayuntamiento de Elche - Ajuntament d'Elx 19 de abril - 6}

Aprobada la reducción de cuotas de las escuelas infantiles municipales.

La comisión de Hacienda modifica los criterios de baremación para dar cabida a un mayor número de familias con necesidades.

También dictamina favorablemente pagos de facturas de 2016 y una modificación presupuestaria para ahorrar en el consumo eléctrico....

Ver más

Ver traducción

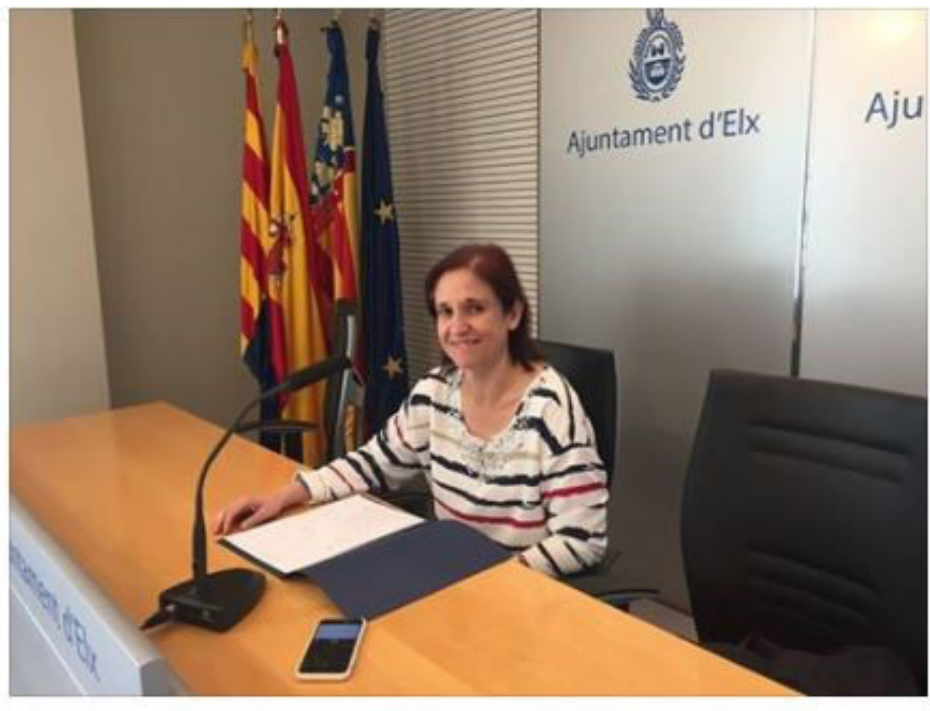

It Me gusta Comentar $\rightarrow$ Compartir

Rocio Juan buenos días. ¿dónde se puede consultar los nuevos criterios de baremación?

Me gusta - Responder - Compartir - 22 de abril a las 9:50

Imagen 9. Publicación de la cuenta y reacción de un usuario. Fuente: Facebook.

Como se puede observar en la imagen 9, un usuario que solicita información no la recibe por parte del Consistorio como sí ocurre en la publicación sobre la bolsa de empleo para enfermeros. Este es un patrón que se repite con frecuencia.

Por tanto, se denota un comportamiento arbitrario y un uso un tanto partidista de la cuenta, la cual responde en función de ciertos intereses del partido que gestiona la cuenta (Sande, 2016).

El comportamiento arbitrario del perfil corporativo@ayuntamientoelche en Facebook va en contra de uno de los principios básicos de un espacio de diálogo social como una página de Facebook de un ente público, el compromiso mutuo, que marca las relaciones entre emisores y receptores donde el mensaje viaja en todas direcciones, es decir, de manera multidireccional (Túñez y Sixto, 2011, p. 3). 


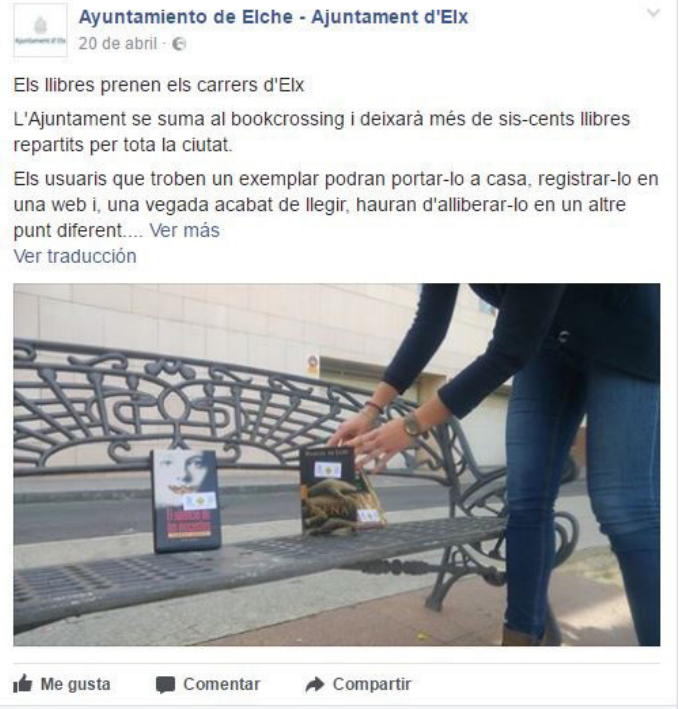

Imagen 10. Publicación de la cuenta el día 20 de abril de 2017. Fuente: Facebook.

Por último, la imagen 10 se corresponde con un post publicado el 20 de abril relacionado con una iniciativa llevada a cabo por la Concejalía de Cultura llamada bookcrossing, que consistió en el reparto de ejemplares de libros por toda la ciudad.

Mónica DG Que idea mas chula para fomentar la lectura y los valores! Cuidar las cosas de los demas, respetar... me parece mmuy buena inniciativa $\because$ Me gusta - Responder - Compartir - (1) 1-20 de abril a las 15:16

Emilieta Payá Bernabéu Ojalá funcione porque es una buena forma de fomentar la lectura que es la mayor fuente de cultura, lo que no se, es si se respetará el tema de la devolución...demasiado gamberro suelto y sería una lástima que se perdieran los libros. Un aplauso a tan bonita iniciativa. Demos un voto de confianza.

Me gusta - Responder - Compartir - (1) 2-21 de abril a las 9:59

Imagen 11. Reacciones positivas de los usuarios al post de la imagen 10.

Fuente: Facebook.

Tal y como podemos observar en la imagen 11, el Ayuntamiento no reacciona ante los comentarios positivos de los usuarios, ya que no ofrece respuesta ni tampoco pulsa cualquiera de los botones que ofrece la red social Facebook.

José Joaquin Belda Gonzalvez Desde luego si se eliminan Carriles Bicis como el de la Universidad y no se señalizan más a se posible por zonas tranquilas no es para animarse Me gusta - Responder - Compartir - 19 de abril a las 18:06

Imagen 12. Selección de comentarios negativos de otros posts del periodo de estudio. Fuente: Facebook. 
En este caso los comentarios presentan un componente negativo, pero al igual que en la imagen anterior, el perfil tampoco reacciona ante las respuestas de los usuarios de la red (ver imagen 12).

Por tanto, la ausencia de respuesta por parte del emisor en la gestión de la publicaciones imposibilita la interacción inmediata, el feedback, que puede contribuir al cambio de actitud en el usuario para, por un lado, cambiar su visión negativa hacia una más positiva o por el contrario, fortalecer la idea favorable inicial que sostiene el seguidor (García Rico, 2015).

\section{Conclusiones}

La investigación señala una tendencia al alza en el uso de los canales sociales de las administraciones locales como medios para la búsqueda y obtención de la información por parte de los seguidores. Sin embargo, el Ayuntamiento no consigue sacar el máximo provecho a sus canales de comunicación y por ello sus cuentas en redes sociales arrojan unos índices de rendimiento de los perfiles inferiores al 50\% en ambos casos. El Departamento de Comunicación debería plantearse la opción de focalizar sus esfuerzos en una única red social como Facebook para atraer toda la fuerza hacia ese perfil y proporcionar una mejor atención a los usuarios, en lugar de usar dos canales distintos tal y como se hace en la actualidad.

Los usuarios se muestran dispuestos a contribuir en la generación de actividad en las cuentas con sus reacciones, sin embargo el Ayuntamiento adopta un comportamiento pasivo que le impide provocar reacciones en los usuarios. Este comportamiento comunicativo del Ayuntamiento denota un interés nulo hacia las opiniones de los ciudadanos y como consecuencia genera un perfil que carece de la actividad e interacción necesaria para la formación de un espacio de diálogo, donde el ciudadano sea el altavoz de las decisiones de la vida pública y donde se produzcan conversaciones enriquecedoras para ambas partes, que sirvan de ayuda a otros usuarios que despierten las mismas dudas o inquietudes.

Por otra parte, los perfiles del Consistorio no consiguen dar el protagonismo necesario a los ciudadanos debido a una actitud distante con ellos. Este comportamiento ha generado una actitud de indiferencia en los seguidores quienes no se sienten partícipes. Por tanto, el Ayuntamiento no se muestra cercano a sus seguidores en las cuentas sociales, aunque cabe señalar un leve compromiso del emisor en la página de Facebook. En conclusión, la gestión comunicativa debe priorizar los intereses de los ciudadanos. Otorgar un estatus de relevancia a los usuarios favorece la generación de relaciones entre emisores y receptores que desembocan en una mejora de las decisiones políticas que afectan a los usuarios.

El perfil de Facebook ha demostrado su capacidad para responder a las dudas de los usuarios y funcionar como un medio por el cual administrar y ampliar información a los seguidores. Sin embargo, este comportamiento se produce de manera arbitraria y en determinadas ocasiones, por lo que se aprecian ciertos intereses de carácter partidista a la hora de gestionar la comunicación. Por ejemplo a la hora de defender el uso de la lengua valenciana.

Se observa un comportamiento comunicativo unidireccional en el perfil de Twitter. La página únicamente cumple la función de transmisora de información a los seguidores, pero no da pie a interactuar con ellos. Todo esto se observa con la utilización de un único hashtag durante el periodo de estudio, el cual no recibió aceptación alguna por parte de los usuarios. 
La intervención escasa del Ayuntamiento en las reacciones de los usuarios no permite al ente público controlar el mensaje comunicativo de los seguidores y ofrecer respuestas que confirmen las opiniones de estos o bien modifiquen sus creencias iniciales. La insuficiente implicación del Ayuntamiento en los temas que preocupan a los ciudadanos genera rumores e informaciones incorrectas. Por tanto, es necesario que el Community Manager responda siempre a los comentarios de los usuarios sean positivos o negativos, a través de respuesta directa o bien con cualquiera de los botones disponibles como el de "Me gusta". Esta actitud contribuirá a la mejora de la percepción de los usuarios sobre la entidad.

A pesar de ofrecer una atención deficiente a los usuarios, el gran volumen de comentarios, compartidos y reacciones que se recogen del estudio de Facebook, muestran la formación de unos usuarios activos que acuden a la página para mostrar sus opiniones tanto positivas como negativas y contribuir a la mejora de la vida pública. Por el contrario en Twitter se observa una menor implicación de ambas partes lo que se genera un espacio sin apenas actividad por parte de los usuarios.

Los perfiles presentan un potencial de influencia elevado debido a la gran cantidad de búsquedas de marca. Al ser un ente público que representa a miles de ciudadanos tiene una gran relevancia en las redes sociales y por ende no necesita una llamada a la acción para conseguir followers.

\section{Bibliografía}

Campillo Alhama, C., y Martínez-Sala, A. M. (2017). Integrated communication 2.0 in municipal administration. El Profesional de la Información, 26 (3): 507-515.

Castelló-Martínez, A., Del Pino-Romero, C., y Ramos-Soler, I. (2014). Twitter como canal de comunicación corporativa y publicitaria.

Criado, J. I., y Rojas, F. (2013). Aproximación general sobre la adopción y uso de las redes sociales en las administraciones públicas. En Criado, J. I., y Rojas, F. (Eds.), Las redes sociales digitales en la gestión y las politicas públicas. Avances y desafíos para un gobierno abierto (pp. 16-31). Barcelona, España: Escola d’Administració Pública de Catalunya.

Criado, J. I., y Rojas Martín, F. (Eds). (2013). Las redes sociales digitales en la gestión y las políticas públicas. Avances y desafíos para un gobierno abierto. Barcelona, España: Escola d’Administració Pública de Catalunya.

Criado, J. Ignacio y Rojas-Martín, Francisco (eds) (2015). Casos de éxito en redes sociales digitales de las administraciones públicas. Barcelona: Escola d'Administració Pública de Catalunya.

De Rosario, A. H., Martín, A. S., y Pérez, M. D. C. C. (2016). The Use of Facebook to Promote Engagement with Local Governments in Spain. In Social Media and Local Governments (pp. 219-241). Springer International Publishing.

Estebaranz, J., y Ramilo, M. (2013). Gobiernos y administraciones locales vascas en las redes sociales. En Criado, J. I., y Rojas, F. (Eds.), Las redes sociales digitales en la gestión y las politicas públicas: avances y desafios para un gobierno abierto (pp. 82-101). Barcelona, España: Escola 
d’Administració Pública de Catalunya.

Fenoll, V., y Cano-Orón, L. (2017). Participación ciudadana en los perfiles de Facebook de los partidos españoles. Análisis de comentarios en la campaña electoral de 2015. Comunicación y Sociedad, 30(4), 131-148.

García Mogedas, C. (2015). Análisis de redes sociales en CB Sevilla y Unicaja de Málaga. Universidad de Sevilla, (49), 20-22. Recuperado https://goo.gl/gFTLb3

García, M. D. L. Á. O., Expósito, M. M., y Verdugo, M. C. (2016). La expansión de las redes sociales. Un reto para la gestión de marketing. Contabilidad y Negocios, 10(20), 59-69.

García Rico, E. (2015). Estudio sobre la comunicación online en redes sociales de Zara, Victoria's Secret y Mango. Universidad de Valladolid (69). Recuperado de https://goo.gl/AFFMlk

Genovès, I. (2013). Prólogo. En Criado, J. I., y Rojas, F. (eds.), Las redes sociales digitales en la gestión y las políticas públicas: avances y desafíos para un gobierno abierto (pp. 10-13). Barcelona, España: Escola d'Administració Pública de Catalunya.

Grande, J. I. C. (2016). Nuevas tendencias en la gestión pública: Innovación abierta, gobernanza inteligente y tecnologías sociales en unas administraciones públicas colaborativas. Herreros, M. C. (2008). La Web 2.0 como red social de comunicación e información/The Web 2.0 as social network of communication and information. Estudios sobre el mensaje periodístico, 14, 345-362.

Merodio, J. (2010). Marketing en Redes Sociales: Mensajes de empresa para gente selectiva. Juan Merodio.

Pereyra Caramé, T. (2012). Las administraciones públicas en las redes sociales. Recuperado de https://goo.gl/7CkE22

Rojas-Martín, F., Criado, J. I. y F. Barrero, D. (2013). "Nace NovaGob, la red social para innovar la administración pública mediante la colaboración de sus profesionales". Revista BoleTIC, diciembre. Recuperado de https://goo.gl/twSSPN

Sánchez, M. M., y Damas, S. H. (2015). Cómo puede contribuir Twitter a una comunicación política más avanzada. Arbor, 191(774), 257.

Sande, P. V. (2016). Usos político-partidistas en cuentas de Twitter de administraciones públicas. Recuperado de https://goo.gl/wJSoCD (Consultado el 2 de marzo de 2017).

Túñez, M., y Sixto, J. (2011). Redes sociales, política y Compromiso 2.0: La comunicación de los diputados españoles en Facebook. de Comunicación Social/08, 85.

Vañó, M. (2015). Estrategias de implantación y gestión de las redes sociales en el sector público. Implantación de un social media plan en la administración pública. En Criado, J. I., y Rojas, F. (eds.), Las redes sociales digitales en la gestión y las políticas públicas: avances y desafíos para un gobierno abierto (pp. 97-113). Barcelona, España: Escola d’Administració Pública de Catalunya. 
MHCJ nº 10 (1) | Año 2019 - Artículo no 3 (138) - Páginas 57 a 81 - mhjournal.org

Villar, J. (2015). Manual de comunicación online de la Concejalia de Comunicación. (Documento inédito). Departamento de Comunicación. Ayuntamiento de Elche.

Vivo, J. M. N. (2010). Redes sociales como paradigma periodístico. Medios españoles en Facebook/Social networks as journalistic paradigm. Spanish media on Facebook. Revista latina de comunicación social, (65), 176-186.

Zumba Campos, A. A. (2015). Manual de uso de las redes sociales para instituciones públicas (Master's thesis, Universidad del Azuay). 
MHCJ no 10 (1) | Año 2019 - Artículo no 3 (138) - Páginas 57 a 81 - mhjournal.org

\section{(c) (i) ()}

Licencia Creative Commons

Miguel Hernández Communication Journal

mhjournal.org

\section{Cómo citar este texto:}

Fernando Miguel Olabe, José Antonio Márquez López (2019): Integración de Twitter y Facebook en la comunicación de la administración local: el Ayuntamiento de Elche como caso de estudio, en Miguel Hernández Communication Journal, nº10 (1), pp. 57 a 81. Universidad Miguel Hernández, UMH (Elche-Alicante). DOI: http://dx.doi.org/10.21134/mhcj.v10i0.275 